PROCEEDINGS OF THE

AMERICAN MATHEMATICAL SOCIETY

Volume 131, Number 4, Pages 1159-1167

S 0002-9939(02)06622-4

Article electronically published on September 19, 2002

\title{
CAPACITY ESTIMATES
}

\author{
DAVID R. ADAMS AND RITVA HURRI-SYRJÄNEN
}

(Communicated by Juha M. Heinonen)

\begin{abstract}
The relationships between certain Sobolev-Orlicz capacities are worked out. This, in particular, determines their null sets.
\end{abstract}

\section{INTRODUCTION}

Several versions of a capacity for the Sobolev-Orlicz space, with the derivatives belonging to the Orlicz space $L^{p}(\log (e+L))^{\theta}$, have recently appeared in literature. A first version appeared in AB94 and then in [Kuz00, and most recently by the authors themselves, AH01]. The purpose of this note is to determine all the relationships between these various versions - this means not only relationships between their null sets, but also any a priori inequalities. These occur as Theorems 2.3 , 3.2 and 3.5. We also attempt to compute, asymptotically, the capacities of a ball $B^{n}\left(x_{0}, r\right) \subset$ Euclidean $\mathbb{R}^{n}$, as a function of $r \rightarrow 0$ (Corollary 2.12). Finally, we prove a simple comparison theorem (Theorem 4.4) which differs from that of AH99, Theorem 5.5.1(d)] in that we do not remain on the critical case unless $\theta=0$.

1.1. Preliminaries. Throughout the paper, the letter $C$ will denote various constants which may differ from one formula to the next even within a single string of estimates. In general we make no attempt to obtain the best values for these constants. The notation $\sim$ means 'is comparable to', i.e. the ratio of the two quantities is bounded above and below by finite positive constants. By $G_{\alpha}$ we denote the Bessel kernel with $\alpha>0$. For the properties of Bessel potentials we refer to AH99, 1.2].

\section{2. $\mathcal{B}_{\alpha, \Phi}(E)$ AND $\mathcal{P}_{\alpha, \Phi}(E)$-CAPACITIES}

The goal is to determine a relationship between the following capacities.

2.1. Definition. Let $\alpha p=n$. For any $E \subset B^{n}\left(0, R_{0}\right)$

$$
\mathcal{B}_{\alpha, \Phi}(E)=\inf \left\{\int \Phi(f) d x: f(x) \geq 0,\left(G_{\alpha} * f\right)(x) \geq 1, x \in E\right\}
$$

Received by the editors July 3, 2001 and, in revised form, November 5, 2001.

2000 Mathematics Subject Classification. Primary 46E35, 31B15.

Key words and phrases. Capacities, Sobolev-Orlicz capacities.

The research of the second author was supported by the Academy of Finland under Grant No. 760095, Grant No. 70063, and Grant No. 42880, by the Väisälä Foundation, and the Finnish Cultural Foundation. 
and

$$
\mathcal{P}_{\alpha, \Phi}(E)=\inf \left\{\|f\|_{L^{\Phi}}: f(x) \geq 0,\left(G_{\alpha} * f\right)(x) \geq 1, x \in E\right\}
$$

where $\|f\|_{L^{\Phi}}<\infty$ is the Luxemburg norm on $L^{\Phi}$, [BS88, Definitions 4.8.2, 4.8.6 and 4.8.10] and $\Phi(t)=t^{p}(\log (e+t))^{\theta}, 0 \leq \theta \leq p-1, \alpha p=n$. We will write $\Phi=\Phi_{p, \theta}$, when $p, \theta$ are fixed.

The main idea is to use the rearrangement equivalent norm for $\|f\|_{L^{\Phi}}$, i.e.

$$
\|f\|_{L^{\Phi}} \sim\left\{\int_{0}^{e^{-2}} f^{*}(t)^{p}\left(\log \frac{1}{t}\right)^{\theta} d t\right\}^{1 / p}
$$

[BS88, Definition 4.6.11, Lemma 4.6.12 and Corollary 1.1.9], and write

$$
\mathcal{P}_{\alpha, \Phi}(E) \sim \inf \left\{\int_{0}^{e^{-2}} f^{*}(t)^{p}\left(\log \frac{1}{t}\right)^{\theta} d t\right\}^{1 / p} .
$$

Also, by [BS88, Proposition 2.1.8], we can write

$$
\mathcal{B}_{\alpha, \Phi}(E) \sim \inf \int_{0}^{e^{-2}} f^{*}(t)^{p}\left(\log \left(e+f^{*}(t)\right)\right)^{\theta} d t
$$

as an alternative description of the capacity $\mathcal{B}_{\alpha, \Phi}(E)$ in terms of the decreasing rearrangement $f^{*}$, that is, a decreasing positive function defined on $\left(0, e^{-2}\right)$, equimeasurable with $f,[\overline{B S 88}$, Definition 2.1.5]. In fact, in [BS88, Proposition 2.1.8] the equivalence is proved only for $\Phi(t)=t^{p}$, but the equivalence follows for $\Phi(t)=t^{p}(\log (e+t))^{\theta}$ in the same way and the upper limit can be chosen to be $e^{-2}$, since we always have $E \subset B^{n}\left(0, R_{0}\right)$ where $B^{n}\left(0, R_{0}\right)$ is a fixed ball.

2.3. Theorem. Let $E \subset B^{n}\left(0, R_{0}\right)$ be any set, $0 \leq \theta \leq p-1$. Then for all $p=n / \alpha>1$, there is a constant $C$ depending only on $n, p$, and $R_{0}$ such that

$$
C^{-1} \mathcal{P}_{\alpha, \Phi}(E)^{p}\left[\log \frac{1}{\mathcal{P}_{\alpha, \Phi}(E)}\right]^{-\theta} \leq \mathcal{B}_{\alpha, \Phi}(E) \leq C \mathcal{P}_{\alpha, \Phi}(E)^{p}
$$

or

$$
C^{-1} \mathcal{B}_{\alpha, \Phi}(E)^{1 / p} \leq \mathcal{P}_{\alpha, \Phi}(E) \leq C \mathcal{B}_{\alpha, \Phi}(E)^{1 / p}\left[\log \frac{1}{\mathcal{B}_{\alpha, \Phi}(E)}\right]^{\theta / p}
$$

whenever $\mathcal{P}_{\alpha, \Phi}(E)<1 / e$.

2.6. Corollary. Capacities $\mathcal{B}_{\alpha, \Phi}$ and $\mathcal{P}_{\alpha, \Phi}$ have the same null sets.

Proof. For Theorem 2.3 we write

$$
\int_{0}^{e^{-2}} f^{*}(t)^{p}\left(\log \frac{1}{t}\right)^{\theta} d t=: N^{p}
$$

and

$$
\int_{0}^{e^{-2}} f^{*}(t)^{p}\left(\log \left(e+f^{*}(t)\right)\right)^{\theta} d t=: M^{p}
$$

C. Bennett and R. Sharpley show that these norms are simultaneously finite, BS88, Lemmata 4.6.12 and 4.6.2], but we need more, namely that one is bounded by a 
constant multiple of the other. As $f^{*}$ is decreasing, we have for all $t \in\left(0, e^{-2}\right)$

$$
\begin{aligned}
f^{*}(t)^{p}\left(\log \frac{1}{t}\right)^{\theta} t & =f^{*}(t)^{p}\left(\log \frac{1}{t}\right)^{\theta} \int_{0}^{t} d s \\
& \leq \int_{0}^{e^{-2}} f^{*}(s)^{p}\left(\log \frac{1}{s}\right)^{\theta} d s=N^{p} .
\end{aligned}
$$

This means

$$
f^{*}(t) \leq \frac{N}{t^{1 / p}}\left(\log \frac{1}{t}\right)^{-\theta / p}
$$

and inserting this into (2.8)

$$
\begin{aligned}
M^{p} & =\int_{0}^{e^{-2}} f^{*}(t)^{p}\left(\log \left(e+f^{*}(t)\right)\right)^{\theta} d t \\
& \leq \int_{0}^{e^{-2}} f^{*}(t)^{p}(\log (2 e))^{\theta} d t+\int_{0}^{e^{-2}} f^{*}(t)^{p}\left(\log \left(2 f^{*}(t)\right)\right)^{\theta} d t \\
& \leq C N^{p}+\int_{0}^{e^{-2}} f^{*}(t)^{p}\left[\frac{1}{p} \log \frac{1}{t}+\log 2 N-\frac{\theta}{p} \log \log \frac{1}{t}\right]^{\theta} \\
& =C N^{p}+\int_{0}^{e^{-2}} f^{*}(t)^{p}\left(\frac{1}{p} \log \frac{1}{t}\right)^{\theta}\left[1+\frac{\log (2 N)^{p}}{\log \frac{1}{t}}-\theta \frac{\log \log \frac{1}{t}}{\log \frac{1}{t}}\right]^{\theta} \leq C N^{p}
\end{aligned}
$$

by (2.7). Hence,

$$
\mathcal{B}_{\alpha, \Phi}(E)^{1 / p} \leq C \mathcal{P}_{\alpha, \Phi}(E) .
$$

Next we find an upper bound for $\mathcal{P}_{\alpha, \Phi}(E)$. Setting $\lambda=\|f\|_{L^{\Phi}}$ where $f$ is a test function for the capacity $\mathcal{P}_{\alpha, \Phi}(E)$, then for $\lambda<1 / e$

$$
\begin{aligned}
1 & \sim \int\left(\frac{f(x)}{\lambda}\right)^{p}\left[\log \left(e+\frac{f(x)}{\lambda}\right)\right]^{\theta} d x \\
& \leq \lambda^{-p} \int f(x)^{p}\left[\log \left(\frac{e+f(x)}{\lambda}\right)\right]^{\theta} d x \\
& \leq C \lambda^{-p}\left(\log \frac{1}{\lambda}\right)^{\theta} \int f^{p}(x) d x+C \lambda^{-p} \int f(x)^{p}(\log (e+f(x)))^{\theta} d x \\
& \leq C \lambda^{-p}\left(\log \frac{1}{\lambda}\right)^{\theta} \int \Phi(f) d x .
\end{aligned}
$$

This implies

$$
\mathcal{P}_{\alpha, \Phi}(E)^{p}\left[\log \frac{1}{\mathcal{P}_{\alpha, \Phi}(E)}\right]^{-\theta} \leq C \lambda^{p}\left(\log \frac{1}{\lambda}\right)^{-\theta} \leq \int \Phi(f) d x,
$$

for arbitrary $f$, and thus

$$
\mathcal{P}_{\alpha, \Phi}(E)\left[\log \frac{1}{\mathcal{P}_{\alpha, \Phi}(E)}\right]^{-\theta / p} \leq C \mathcal{B}_{\alpha, \Phi}(E)^{1 / p} .
$$

Hence, combining with (2.9) yields

$$
C^{-1} \mathcal{B}_{\alpha, \Phi}(E)^{1 / p} \leq \mathcal{P}_{\alpha, \Phi}(E) \leq C \mathcal{B}_{\alpha, \Phi}(E)^{1 / p}\left[\log \frac{1}{\mathcal{B}_{\alpha, \Phi}(E)}\right]^{\theta / p}
$$


for all $p>1$. On the other hand, (2.9) and (2.10) directly imply

$$
C^{-1} \mathcal{P}_{\alpha, \Phi}(E)^{p}\left[\log \frac{1}{\mathcal{P}_{\alpha, \Phi}(E)}\right]^{-\theta} \leq \mathcal{B}_{\alpha, \Phi}(E) \leq C \mathcal{P}_{\alpha, \Phi}(E)^{p}
$$

for all $p>1$.

Now we calculate the capacity $\mathcal{P}_{\alpha, \Phi}\left(B^{n}\left(x_{0}, r\right)\right)$.

2.11. Theorem. Let $B^{n}\left(x_{0}, r\right) \subset B^{n}\left(0, R_{0}\right)$ be a ball with a center $x_{0}$ and radius $r>0$. Let $p=n / \alpha>1$. Then for sufficiently small $r$

$$
\mathcal{P}_{\alpha, \Phi_{p, p-1}}\left(B^{n}\left(x_{0}, r\right)\right) \sim\left(\log \log \frac{1}{r}\right)^{(1-p) / p}
$$

and

$$
\mathcal{P}_{\alpha, \Phi_{p, \theta}}\left(B^{n}\left(x_{0}, r\right)\right) \sim\left(\log \frac{1}{r}\right)^{(1-p+\theta) / p}
$$

when $0 \leq \theta<p-1$.

Proof. If $\Phi(t)=t^{p}(\log (e+t))^{\theta}, \alpha p=n$, then $\tilde{\Phi}(t) \sim t^{p^{\prime}}(\log (e+t))^{-\theta p^{\prime} / p}, p^{\prime}=$ $p /(p-1)$, is the conjugate Young function. We want to obtain an upper bound on $\left\|I_{\alpha} * \mu\right\|_{L^{\tilde{\Phi}}}$ with $d \mu=\chi_{B_{r}} d x$, where $B_{r}=B^{n}(0, r)$ and $I_{\alpha} \sim|x|^{\alpha-n}$ is the Riesz kernel. From [BS88, Definition 4.6.11, Lemma 4.6.12 and Corollary 1.1.9]

$$
\left\|I_{\alpha} * \chi_{B_{r}}\right\|_{L^{\tilde{\Phi}}} \sim\left[\int_{0}^{1 / 2}\left(\left(I_{\alpha} * \chi_{B_{r}}\right)^{*}(t)\right)^{p^{\prime}}\left(\log \frac{1}{t}\right)^{-\theta p^{\prime} / p} d t\right]^{1 / p^{\prime}} .
$$

By definition $f^{* *}(t)=\frac{1}{t} \int_{0}^{t} f^{*}(s) d s$. By (1.8.10) in [Zie89] and O'Neil's inequality, [ie89. Lemma 1.8.8],

$$
\left(I_{\alpha} * \chi_{B_{r}}\right)^{*}(t) \leq\left(I_{\alpha} * \chi_{B_{r}}\right)^{* *}(t)=t I_{\alpha}^{* *}(t) \chi_{B_{r}}^{* *}(t)+\int_{t}^{\infty} I_{\alpha}^{*}(s) \chi_{B_{r}}^{*}(s) d s .
$$

Since $I_{\alpha}^{*}(t) \sim I_{\alpha}^{* *}(t) \sim t^{\alpha / n-1}$,

$$
\chi_{B_{r}}^{*}(t) \sim \begin{cases}1, & t \leq r^{n}, \\ 0, & \text { otherwise },\end{cases}
$$

and

we have

$$
\chi_{B_{r}}^{* *}(t) \sim \begin{cases}\frac{r^{n}}{t}, & t>r^{n} \\ 1, & t \leq r^{n}\end{cases}
$$

$$
\begin{gathered}
\left(I_{\alpha} * \chi_{B_{r}}\right)^{*}(t), \\
\leq C \begin{cases}t^{\alpha / n-1} r^{n}, & t>r^{n}, \\
r^{\alpha}, & t \leq r^{n} .\end{cases}
\end{gathered}
$$

Let $\Phi(t)=\Phi_{p, \theta}(t)$ and $\tilde{\Phi}(t)=\Phi_{p^{\prime},-\theta p^{\prime} / p}(t)$ with $0 \leq \theta<p-1$. Hence

$$
\begin{aligned}
\left\|I_{\alpha} * \chi_{B_{r}}\right\|_{L^{\tilde{\Phi}}}^{p^{\prime}} & \sim \int_{0}^{r^{n}} r^{\alpha p^{\prime}}\left(\log \frac{1}{t}\right)^{-\theta p^{\prime} / p} d t+r^{n p^{\prime}} \int_{r^{n}}^{1 / 2} t^{(\alpha / n-1) p^{\prime}}\left(\log \frac{1}{t}\right)^{-\theta p^{\prime} / p} d t \\
& \sim r^{\alpha p^{\prime}}\left(\log \frac{1}{n}\right)^{-\theta p^{\prime} / p} r^{n}+\left(\log \frac{1}{r}\right)^{1-\theta p^{\prime} / p} r^{n p^{\prime}} \leq C\left(\log \frac{1}{r}\right)^{1-\theta p^{\prime} / p} r^{n p^{\prime}} .
\end{aligned}
$$


Note that from the work of N. Aissaoui and A. Benkirane, Ais95], [AB94, we have

$$
\mathcal{P}_{\alpha, \Phi}(E)=\sup \left\{\|\nu\|: \operatorname{spt} \nu \subset E \text { and }\left\|G_{\alpha} * \nu\right\|_{L^{\tilde{\Phi}}} \leq 1\right\},
$$

for all $E \subset B^{n}\left(0, R_{0}\right)$. So set

$$
d \nu=\frac{\chi_{B_{r}} d x}{r^{n}\left(\log \frac{1}{r}\right)^{1 / p^{\prime}-\theta / p}}
$$

then $\left\|I_{\alpha} * \nu\right\|_{L^{\tilde{\Phi}}} \leq 1$ implies

$$
\mathcal{P}_{\alpha, \Phi}\left(B^{n}\left(x_{0}, r\right)\right) \geq C\left(\log \frac{1}{r}\right)^{-1 / p^{\prime}+\theta / p} .
$$

Next, we obtain an upper bound

$$
\mathcal{P}_{\alpha, \Phi}\left(B^{n}\left(x_{0}, r\right)\right) \leq C\left(\log \frac{1}{r}\right)^{-1 / p^{\prime}+\theta / p}
$$

by choosing $f(y)=|y|^{-\alpha}\left(\log \frac{1}{|y|}\right)^{-\theta p^{\prime} / p}$ when $2 r<|y|<1 / 2$ and $f(y)=0$ otherwise. When $|x|<r$ and $r<|y| / 2$, then $|x-y| \geq|y| / 2$, and

$$
\begin{aligned}
\left(I_{\alpha} * f\right)(x) & \sim \int_{2 r<|y|<1 / 2}|x-y|^{\alpha-n}|y|^{-\alpha}\left(\log \frac{1}{|y|}\right)^{-\theta p^{\prime} / p} d y \\
& \leq C \int_{2 r<|y|<1 / 2}|y|^{\alpha-n}|y|^{-\alpha}\left(\log \frac{1}{|y|}\right)^{-\theta p^{\prime} / p} d y \\
& \leq C \int_{r}^{1 / 2}\left(\log \frac{1}{\rho}\right)^{-\theta p^{\prime} / p} \frac{d \rho}{\rho} \leq C\left(\log \frac{1}{r}\right)^{1-\theta p^{\prime} / p} .
\end{aligned}
$$

So, when $\alpha p=n$,

$$
\begin{aligned}
\|f\|_{L^{\Phi}}^{p} & \leq C \int_{2 r}^{1 / 2}\left[t^{-\alpha}\left(\log \frac{1}{t}\right)^{-\theta p^{\prime} / p}\right]^{p}\left(\log \frac{1}{t}\right)^{\theta} t^{n} \frac{d t}{t} \\
& \sim \int_{2 r}^{1 / 2}\left(\log \frac{1}{t}\right)^{\theta\left(1-p^{\prime}\right)} \frac{d t}{t} \\
& \sim\left(\log \frac{1}{r}\right)^{1+\theta\left(1-p^{\prime}\right)} .
\end{aligned}
$$

Thus, for a ball $B^{n}\left(x_{0}, r\right)$,

$$
\mathcal{P}_{\alpha, \Phi}\left(B^{n}\left(x_{0}, r\right)\right) \leq C\left(\log \frac{1}{r}\right)^{\theta p^{\prime} / p-1}\|f\|_{L^{\Phi}} \leq C\left(\log \frac{1}{r}\right)^{(1-p+\theta) / p} .
$$

The case $\theta=p-1$ is similar, giving the double logarithm.

From Theorems 2.3 and 2.11, we have

2.12. Corollary. Let $B^{n}\left(x_{0}, r\right) \subset B^{n}\left(0, R_{0}\right)$ be a ball with sufficiently small radius $r>0$, and $p=n / \alpha>1$. Then there is a constant $C$ depending only on $n, p$ and $R_{0}$ such that

$$
C^{-1}\left(\log \log \frac{1}{r}\right)^{1-p}\left(\log \log \log \frac{1}{r}\right)^{1-p} \leq \mathcal{B}_{\alpha, \Phi_{p, p-1}}\left(B^{n}\left(x_{0}, r\right)\right) \leq C\left(\log \log \frac{1}{r}\right)^{1-p}
$$

and

$$
C^{-1}\left(\log \frac{1}{r}\right)^{1-p+\theta}\left(\log \log \frac{1}{r}\right)^{-\theta} \leq \mathcal{B}_{\alpha, \Phi_{p, \theta}}\left(B^{n}\left(x_{0}, r\right)\right) \leq C\left(\log \frac{1}{r}\right)^{1-p+\theta}
$$

for $0 \leq \theta<p-1$. 
2.13. Remark. For a ball $B^{n}\left(x_{0}, r\right) \subset B^{n}\left(0, R_{0}\right)$ with sufficiently small $r$, by [AH01. Theorem 3.1] we have $\mathcal{B}_{1, \Phi_{n, n-1}}\left(B^{n}\left(x_{0}, r\right)\right) \sim\left(\log \log \frac{1}{r}\right)^{1-n}$ and $\mathcal{B}_{1, \Phi_{n, \theta}}\left(B^{n}\left(x_{0}, r\right)\right)$ $\sim\left(\log \frac{1}{r}\right)^{1-n+\theta}$ for $0 \leq \theta<n-1$. A similar result for the higher order case is unknown. Note that notation in AH01] is different.

\section{KUZNETSOV'S CAPACITY}

In the paper by S. Kuznetsov, Kuz00, concerning removable singularities for $L(u)=\Phi(u)$, where $L$ is a second order elliptic differential operator and $\Phi$ is an increasing convex function with $\Phi(0)=0$, the capacity with $\alpha=2$ is

$$
b_{\alpha, \tilde{\Phi}}(E)=\sup \left\{\|\nu\|: \operatorname{spt} \nu \subset E \text { and } \int \tilde{\Phi}\left(G_{\alpha} * \nu\right) \leq 1\right\} ;
$$

here $\Phi$ and $\tilde{\Phi}$ are conjugate Young functions.

3.1. Remark. If we define

$$
\mathcal{K}_{\alpha, \Phi}(E)=\inf \left\{\|f\|_{L^{\Phi}}: f \geq 0 \text { and } I_{\alpha} * f \geq 1 \text { on } E\right\}
$$

and

$$
R_{\alpha, \Phi}(E)=\inf \left\{\int \Phi(f) d x: f \geq 0 \text { and } I_{\alpha} * f \geq 1 \text { on } E\right\},
$$

where $I_{\alpha}$ is the Riesz kernel with $0<\alpha<n$, then

$$
\mathcal{K}_{\alpha, \Phi}(E) \sim \mathcal{P}_{\alpha, \Phi}(E)
$$

and

$$
R_{\alpha, \Phi}(E) \sim \mathcal{B}_{\alpha, \Phi}(E)
$$

for all sets $E \subset B^{n}\left(0, R_{0}\right)$ where $B^{n}\left(0, R_{0}\right)$ is a fixed ball.

From the work of N. Aissaoui and A. Benkirane, Ais95, [AB94,

$$
\mathcal{P}_{\alpha, \Phi}(E)=\sup \left\{\|\nu\|: \operatorname{spt} \nu \subset E \text { and }\left\|G_{\alpha} * \nu\right\|_{L^{\tilde{\Phi}}} \leq 1\right\},
$$

for all $E \subset B^{n}\left(0, R_{0}\right)$. This leads to

3.2. Theorem. Let $E \subset B^{n}\left(0, R_{0}\right)$. Then

$$
b_{\alpha, \tilde{\Phi}}(E) \sim \mathcal{P}_{\alpha, \Phi}(E) .
$$

Proof. The argument is similar to that of Theorem 2.3 but we provide the details for the convenience of the reader. We have $\tilde{\Phi}(t) \sim t^{p^{\prime}}(\log (e+t))^{-\theta p^{\prime} / p}$ as $t \rightarrow \infty$; $p^{\prime}=p /(p-1)$. By (2.2), $\left\|I_{\alpha} * \nu\right\|_{L^{\tilde{\Phi}}} \leq 1$ yields

$$
C\left(\left[\left(I_{\alpha} * \nu\right)^{*}(t)\right]^{p^{\prime}}\left(\log \frac{1}{t}\right)^{-\theta p^{\prime} / p} t\right)^{1 / p^{\prime}} \leq\left\|I_{\alpha} * \nu\right\|_{L^{\tilde{\Phi}}} \leq 1 .
$$

If we write $\left\|I_{\alpha} * \nu\right\|_{L^{\tilde{\Phi}}}=N^{*}$, then

$$
\left(I_{\alpha} * \nu\right)^{*}(t) \leq \frac{C N^{*}}{t^{1 / p^{\prime}}}\left(\log \frac{1}{t}\right)^{\theta / p} .
$$


Hence, for $N^{*} \leq 1$,

$$
\begin{aligned}
\int \tilde{\Phi}\left(I_{\alpha} * \nu\right) d x & \sim \int_{0}^{1}\left[\left(I_{\alpha} * \nu\right)^{*}(t)\right]^{p^{\prime}}\left[\log \left(e+\left(I_{\alpha} * \nu\right)^{*}(t)\right)\right]^{-\theta p^{\prime} / p} d t \\
& \geq \int_{0}^{1}\left[\left(I_{\alpha} * \nu\right)^{*}(t)\right]^{p^{\prime}}\left[\frac{1}{p^{\prime}} \log \frac{1}{t}-\log \frac{1}{N^{*}}+\frac{\theta}{p} \log \log \frac{1}{t}\right]^{-\theta p^{\prime} / p} d t \\
& \geq \int_{0}^{1}\left[\left(I_{\alpha} * \nu\right)^{*}(t)\right]^{p^{\prime}}\left(\log \frac{1}{t}\right)^{-\theta p^{\prime} / p}\left[\frac{1}{p^{\prime}}+\frac{\theta}{p} \frac{\log \log \frac{1}{t}}{\log \frac{1}{t}}\right]^{-\theta p^{\prime} / p} d t \\
& \geq C\left\|I_{\alpha} * \nu\right\|_{L^{\tilde{\Phi}}}^{p^{\prime}}
\end{aligned}
$$

Thus

$$
\int \tilde{\Phi}\left(I_{\alpha} * \nu\right) \geq C|| I_{\alpha} * \nu \|_{L^{\tilde{\Phi}}}^{p^{\prime}},
$$

and so

$$
\mathcal{P}_{\alpha, \Phi}(E) \geq C b_{\alpha, \tilde{\Phi}}(E) .
$$

To go the other way: Let $\lambda=\|g\|_{L^{\tilde{\Phi}}}$,

$$
\int\left(\frac{g}{\lambda}\right)^{p^{\prime}}\left[\log \left(e+\frac{g}{\lambda}\right)\right]^{-\theta p^{\prime} / p} d x \leq C .
$$

To have

$$
\left(\log \frac{1}{\lambda}\right)^{-\theta p^{\prime} / p} \leq 1
$$

we assume $\lambda \leq e^{-1}$. Hence,

$$
\begin{aligned}
C & \geq \int\left(\frac{g}{\lambda}\right)^{p^{\prime}}\left[\log \left(e+\frac{g}{\lambda}\right)\right]^{-\theta p^{\prime} / p} d x \\
& \geq \int\left(\frac{g}{\lambda}\right)^{p^{\prime}}\left[\log \left(\frac{e+g}{\lambda}\right)\right]^{-\theta p^{\prime} / p} d x \\
& =\lambda^{-p^{\prime}}\left(\log \frac{1}{\lambda}\right)^{-\theta p^{\prime} / p} \int g^{p^{\prime}}\left[\frac{\log (e+g)}{\log \frac{1}{\lambda}}+1\right]^{-\theta p^{\prime} / p} d x \\
& \geq C \lambda^{-p^{\prime}}\left(\log \frac{1}{\lambda}\right)^{-\theta p^{\prime} / p} \int g^{p^{\prime}}[\log (e+g)]^{-\theta p^{\prime} / p} d x
\end{aligned}
$$

Thus

$$
\int \tilde{\Phi}(g) d x \sim \int g^{p^{\prime}}(\log (e+g))^{-\theta p^{\prime} / p} d x \leq C \lambda^{p^{\prime}}\left(\log \frac{1}{\lambda}\right)^{\theta p^{\prime} / p} .
$$

So if $g=\left(I_{\alpha} * \nu\right)$, then

$$
\int \tilde{\Phi}\left(I_{\alpha} * \nu\right) d x \leq C\left\|I_{\alpha} * \nu\right\|_{L^{\tilde{\Phi}}}^{p^{\prime}}\left(\log \frac{2}{\left\|I_{\alpha} * \nu\right\|_{L^{\tilde{\Phi}}}}\right)^{\theta p^{\prime} / p} .
$$

Let $\nu$ be the capacity extremal for $\mathcal{P}_{\alpha, \Phi}$, i.e. the measure that gives equality

$$
\|\nu\|=\mathcal{P}_{\alpha, \Phi}(E) .
$$

For such a measure, one must have

$$
\left\|I_{\alpha} * \nu\right\|_{L^{\tilde{\Phi}}}=1 .
$$


Thus, for $\mu=C \nu$ with sufficiently small $C>0$, we have

$$
\int \tilde{\Phi}\left(I_{\alpha} * \mu\right) d x \leq C \|\left. I_{\alpha} * \mu\right|_{L^{\tilde{\Phi}}} ^{p^{\prime}}\left(\log \frac{2}{\left\|I_{\alpha} * \mu\right\|_{L^{\tilde{\Phi}}}}\right)^{\theta p^{\prime} / p} \leq 1,
$$

so

$$
b_{\alpha, \tilde{\Phi}}(E) \geq\|\mu\| \geq C \mathcal{P}_{\alpha, \Phi}(E) .
$$

Hence, by (3.3) and (3.4)

$$
b_{\alpha, \tilde{\Phi}}(E) \sim \mathcal{P}_{\alpha, \Phi}(E)
$$

where $\Phi$ and $\tilde{\Phi}$ are conjugate Young functions.

Thus combining Theorems 2.3 and 3.2

3.5. Theorem. Let $E \subset B^{n}\left(0, R_{0}\right)$ and $\alpha p=n$. Then

$$
\mathcal{B}_{\alpha, \Phi}(E)^{1 / p} \leq C b_{\alpha, \tilde{\Phi}}(E) .
$$

The reverse inequality is well known when $\theta=0$, but open for $\theta>0$.

\section{A comparison theorem}

For comparison results to $b_{\alpha, \Phi_{p, \theta}}$ with different values of $\alpha, p$ and $\theta$, a Sobolev inequality for the function $\Phi(t)=t^{r}(\log (e+t))^{\eta}, \alpha r=n, 0 \leq \eta \leq r-1$, is needed. We state the Sobolev inequality which might be of independent interest.

4.1. Theorem. Let $r^{*}=\frac{n r}{n-\alpha r}$ with $\alpha r<n$ and let $0 \leq \eta \leq r-1$.Then

$$
\left\|G_{\alpha} * f\right\|_{L^{r^{*}}(\log (e+L))^{\eta^{*} / r}} \leq C\|f\|_{L^{r}(\log (e+L))^{\eta}} .
$$

The inequality follows by using Hedberg's method, Hed72. Also, it agrees with the Orlicz-Sobolev inequality of R. A. Adams, Ada75, when $\alpha$ is a positive integer.

4.3. Remark. We agree on the following notation $b_{\alpha, p ; \theta}(E)=b_{\alpha, \Phi}(E)$ when $\Phi(t)=$ $\Phi_{p, \theta}(t)=t^{p}(\log (e+t))^{\theta}$.

4.4. Theorem. Let $E \subset B^{n}\left(0, R_{0}\right)$. Let $\alpha>\beta, 1<p<q, \alpha p=\beta q=n$, and $0 \leq \theta \leq p-1$. Then

$$
b_{\beta, q ; \theta}(E) \leq C b_{\alpha, p ; \frac{\theta_{p}}{q}}(E) .
$$

Proof. The dual space for $L^{p}(\log (e+L))^{\delta}$ is $L^{p^{\prime}}(\log (e+L))^{-\delta /(p-1)}$ and the dual space for $L^{q}(\log (e+L))^{\theta}$ is $L^{q^{\prime}}(\log (e+L))^{-\theta /(q-1)}$. Thus by (4.2), we have for any measure $\nu$

$$
\begin{aligned}
& \left\|G_{\alpha} * \nu\right\|_{L^{\left(q^{\prime}\right) *}(\log (e+L))^{-\theta\left(q^{\prime}\right) * /\left((q-1) q^{\prime}\right)}}
\end{aligned}
$$

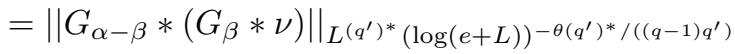

$$
\begin{aligned}
& \leq C|| G_{\beta} * \nu \|_{L^{q^{\prime}}(\log (e+L))^{-\theta /(q-1)}} .
\end{aligned}
$$

Since $\alpha p=\beta q=n$, we get $\left(q^{\prime}\right)^{*}=p^{\prime}$. Here, in order to get $\delta$ in terms of $p, q, \theta$, we set $-\frac{\delta}{p-1}=-\frac{\theta p^{\prime}}{(q-1) q^{\prime}}$, which implies $\delta=\frac{\theta p}{q}$. Thus, by Theorem 3.2

$$
b_{\beta, q ; \theta}(E) \leq C b_{\alpha, p ; \frac{\theta p}{q}}(E)
$$

for some constant independent of $E$. 
4.5. Remark. Now since $\alpha$-potentials of functions in $L^{p}(\log (e+L))^{\theta}$ with $\alpha p=n$ spaces are only embedded in the continuous functions when $\theta>p-1$ and not when $\theta \leq p-1$, we notice that the capacity at the extreme case does not dominate another such capacity, i.e.

$$
b_{\beta, q ; p^{\prime}}(E) \leq C b_{\alpha, p ; p-1}(E),
$$

and $\frac{q}{p^{\prime}}<q-1$, since $q>p$. Thus the right side of (4.6), at the critical case $p-1$, does not dominate the critical case $q-1$ on the left-hand side. Hence, one stays on the critical case only when $\theta=0$, [AH99, Theorem 5.5.1. (d)].

\section{REFERENCES}

[AB94] N. Aissaoui and A. Benkirane, Capacités dans les espaces d'Orlicz, Ann. Sci. Math. Québec 18 (1994), 1-23. MR 95h:31009

[Ada75] R. A. Adams, Sobolev Spaces, Academic Press, New York, 1975, Series in Pure and Applied Mathematics \#65. MR 56:9247

[AH99] D. R. Adams and L. I. Hedberg, Function Spaces and Potential Theory, Springer-Verlag, Berlin Heidelberg New York, 1996, Grundlehren der mathematischen Wissenschaften 314. MR 97j:46024

[AH01] D. R. Adams and R. Hurri-Syrjänen, Vanishing exponential integrability for functions whose gradients belong to $L^{n}(\log (e+L))^{\alpha}$, To appear in J. Funct. Anal. (2001).

[Ais95] N. Aissaoui, Note sur la capacitabilité dans les espaces d'Orlicz, Ann. Sci. Math. Québec 19 (1995), 107-113. MR 96i:31007

[BS88] C. Bennett and R. Sharpley, Interpolation of Operators, Academic Press, Inc., New York, 1988. MR 89e:46001

[Hed72] L. I. Hedberg, On certain convolution inequalities, Proc. Amer. Math. Soc. 36 (1972), 505-510. MR 47:794

[Kuz00] S. E. Kuznetsov, Removable singularities for $L u=\psi(u)$ and Orlicz capacities, J. Funct. Anal. 170 (2000), 428-449. MR 2001g:35078

[Zie89] W. P. Ziemer, Weakly Differentiable Functions, Springer-Verlag, New York, NY, 1989. MR 91e:46046

Department of Mathematics, University of Kentucky, 715 Patterson Office Tower, LeXington, KentuCKY 40506-0027

E-mail address: dave@ms.uky.edu

Department of Mathematics, P.O. Box 4 (Yliopistonkatu 5), Fin-00014 University of HeLsinki, Finland

E-mail address: hurri@ms.uky.edu

E-mail address: ritva.hurri-syrjanen@helsinki.fi 